\title{
Current Research Trends in Sound Transmission Loss of Silencers
}

\author{
Nouby M. Ghazaly and Ahmad O. Moaaz \\ Mechanical Engineering Dept., Faculty of Engineering, South Valley University, Qena-83523, Egypt. \\ Mechanical Engineering Dept., Faculty of Engineering, Beni-Suef University,BeniSuef -62511, Egypt.
}

\begin{abstract}
In recent years, the influence of automobile emissions and noise on the environment has become an increasingly serious problem. Particularly, exhaust system is known to be a predominant component of the automobile emissions and noise. Fortunately, over the last few decades, engine exhaust noise is controlled through the use of silencers or mufflers. The analysis of such complex mufflers has always been a great challenge and an active area of research in recent years. For a suitable design of mufflers, there are two main categories that are used to study this problem: the Transmission Loss (TL) and Insertion Loss (IL). Currently the transmission lossis preferred and widely used for determining muffler performance. This paper aims to present an updated review of papers, conference papers, books, dissertations dealing with muffler acoustics performance using transmission loss.
\end{abstract}

Keywords:Automobile Emissions and Noise, Silencers or Mufflers, Transmission Loss.

\section{Introduction}

Noise from the automobile is generated by several sources such as the internal combustion engine, the brake system, the inlet and exhaust system and the flow around the body of the automobile. The authors paid attention to the exhaust system which is believed to be one of the main factors of noise pollution of the urban environment. Intake and exhaust system noise draws a huge contribution to the interior and exterior noise of cars. A major factor in engine and vehicle emitted noise is the exhaust system. With regard to the sound pressure levels, the untreated exhaust noise is often ten times greater than all the structural noise of the system combined [1]. Thus, there has been a great deal of research and development in the area of predicting muffler performance $[2: 7]$.

Muffler performance from a designer's standpoint is characterized by either the Transmission Loss (TL) or Insertion Loss (IL) of the muffler. Transmission Loss is the ratio of the sound power of the incident (progressive) pressure wave at the inlet of the muffler to the sound power of the transmitted pressure wave at the outlet of the muffler [8]. The benefit of TL is that it is a parameter of the muffler alone and the source or termination properties are not needed. Because of the simplifications, the TL is the most common parameter for muffler performance. Insertion loss is defined as the difference between the acoustic pressure radiated from the source with and without the muffler attached [8]. The IL is a more accurate method of determining muffler performance as it is measured in actual operating conditions with the source properties applied.

The commercial automotive mufflers are generally of a complicated shape with multiply connected parts and complex acoustic elements. Typically mufflers are classified under two different categories, dissipative and reflective [9]. A dissipative muffler is based on the principle of converting the exhaust noise energy caused by fluctuating pressure waves into heat. This is done using sound-absorbing materials such as porous sound attenuating woven fibers and perforated tubing. Another benefit of dissipative mufflers is that the pressure drop across the system is relatively low because the flow path is not significantly altered by flow reversals, twists and turns, or by any other means. Reactive silencers generally consist of a number of chambers and tubes. Those configurations can provide effective noise attenuation at lower frequencies, but fail to attenuate higher-frequency noise and usually produce a high pressure drop due to the presence of baffles and flow reversals [10].

In this review paper, several state of the art papers and even books on muffler performance noise have been presented. Many of them have analytically and sharply accounted for the impressive amount of research undertaken on this topic. This state of the art review focuses on the still-open questions that appear crucial from the perspective of a leading muffler manufacturer. The paper deals with the phenomena of muffler performance noise, the experimental and theoretical methods for studying such phenomena, and the actions that are identified to be necessary to definitely solve the addressed problem.

\section{Transmission Loss (TL)}

Accurate prediction of sound radiation characteristics from reactive mufflers is of significant importance in automotive exhaust system design. The most commonly used parameter to evaluate the sound 
radiation characteristics of muffler is sound transmission loss (TL) [11:18]. Transmission loss is one of the most frequently used criteria of muffler performance because it can be predicted very easily from the known physical parameters of the muffler. Many tools are available to simulate the transmission loss characteristics of a muffler. They vary in terms of complexity and assumptions. However analytical models if developed give accurate results especially in low and mid frequency region in no time by directly applying the developed formula. Such a developed formula can be very useful to represent performance characteristics of the muffler for relative comparison of design alternatives at design stage.Parrot et al, 1973[19] improved a standard transmission line theory for designing an expansion chamber muffler as an application of helicopter. A computerized method using EXRSIL and FORTRAN was used. They conducted an optimization procedure that adjusts muffler component lengths to achieve minimum transmission loss over a specified frequency range. The results showed that three-stage expansion chamber muffler together for the exhaust pipe-Y-connector for combining the exhaust gases from all cylinders reduced the exhaust noise and no significant engine performance loss.

Amiya and Mohanty, in 1993[20] carried out experimental and numerical investigations for passive mufflers. A multi domain boundary element method (BEM) works as a numerical techniques for modeling such mufflers and predicting (TL). To successfully incorporate perforates and sound absorbing materials in boundary element models, experiments were conducted to determine the perforate transfer impedance and the propagation constant. TL of a reactive and dissipative mufflers obtained using an analytical one-dimensional solution, thus BEM validated. They discussed various techniques to determine TL. It was found that an excellent results were found between experimental and boundary element method.

Development a formula for predicting transmission loss (TL) of a long rigid duct was conducted by Chen et al, 1998 [12]. By adding some improvements like Helmholtz resonator, experimentally great results were obtained. They concluded that the acoustic interaction between two acoustic filters can be ignored if the distance between them is great enough.In the previous work by Yeh et al. 2003 [21], the graphical analysis of optimal shape designs was discussed in order to improve the performance of sound transmission loss (TL) on a constrained single expansion muffler. Typically mufflers are classified under two different categories, dissipative and reflective.Moreover, Yeh et al, 2004 [22] Presented optimal design of a single chamber muffler with side inlet/outlet. They described both the graphic analysis and the computer aided numerical assessment. They obtained the results and then verify them with Kuhn-tucker condition for accuracy. The simulated results showed that STL of muffler is exactly maximized at the desired frequency.

Investigation of the acoustic performance of a single pass perforated silencer through experimentally, analytically and BEM study of a hybrid concept was examined by Selamet et al. 2003[23]. A single-chamber dissipative silencer tested varying the outer diameter and material density. They observed that increase in density increasing resonance frequency as well the transmission loss increased too. Lower resonance frequency decreased again by changing outer diameter up, but unlike reactive chambers may not increase peak magnitude of TL. It was also found that three dimensional BEM showed an agreement with experimental results. Reactive element combined with two dissipative chambers increase TL leading to effective hybrid silencer. The effects of partial fiber filling, location, and reactive chamber shape and perforations distribution remain to be investigated. Also, Tao and Syebert, 2003 [24] Discussed two measurements that don't require anechoic termination. The methods were the two load and two source methods, both were demonstrated on two muffler types, simple and double expansion chambers. The measured TL compared with results obtained from BEM results. The measured TL of both methods proves that TL can be determined reliably without an anechoic termination.

For the transmission loss measurement in presence of flow Hyunsu Kim,2011[16] developed flow impedance tube setup using burst signals. Incorporation of burst-signal separation with a long tube, and so by separating transmitted and reflected waves in time domain, so an anechoic termination on the downstream side was achieved, by applying wave decomposition in frequency domain on the upstream side, incident waves of burst signal obtained. Microphone calibration, time domain editing functions and hydraulic losses were discussed to improve the accuracy or the capacity of the transmission loss setup in the presence of flow. It was found that in the presence of flow, the acoustic performance of a Helmholtz resonator change dramatically with an increase in the peak transmission loss frequency and a decrease in the magnitude.

Acoustic diffraction by a muffler contains perforated ducts in 3-D case was investigated by Besache et al, 2006[25]. A harmonic time regime and stationary fluid Helmholtz equation was solved in 3-D. To use FEM two fictitious boundaries bound the domain where set transparent boundary condition called radiation condition at finite distance. They tested two models of exhaust systems and compared FEM results with experimental and transfer matrix method (TMM) numerical results. It was found that the FEM results are better, while the peaks of TL curve by FEM were with small translation.In addition, Mihai Bugaru and OvidueVasili, 2007 [26] Modeled mufflers on base of the transfer matrix method (TMM). Theyreported that TMM could be offered fast prototype solution for muffler designers. They also presented an overview of the principles of TMM predicts the transmission loss TL of a muffler. It could be concluded that the TMM is an effective tool in order to estimate 
the TL of a muffler in the design early stage taking into account the specific constructive elements of the muffler.

Shape optimization on mufflers within a limited space is essential for industry where the equipment layout is occasionally tight and the available space for a muffler is limited for maintenance and operation purposes. To proficiently enhance the acoustical performance within a constrained space, the selection of an appropriate acoustical mechanism and optimizer becomes crucial.An optimization of the acoustic performance in low cost, simple geometry mufflers using micro perforated panels (MPP)was carried out by Florent Masson et al, 2008 [17]. They compared TL given by a computed model with laboratory measurements both for mufflers contain MPP and without. It was observed that MPPs could be an alternative to improve the TL for low frequencies. The optimization based on easy TMM. BEM was used to compare the evaluation of TL. Different configurations have been tested to detect the real effect of resonator absorbers based on MPP in the expansion chambers.Rahul Bansal, 2005[27] Investigated acoustic properties of perforates chattered in a duct through experiments random sound. Two microphones in two points used to measure sound pressure intensity in the tube. The auto and cross spectra signal obtained by two microphones are used to measure the intensity of incident and reflected wave by decomposition and the phase angle between the two. By decomposition the reflected and incident wave's acceptable results achieved comparing with other theoretical techniques models. It is reported that the acoustic properties found can be used to improve performance corresponding to sound of a system.In 2005, Potente and Daniel [8] discussed the basic types of mufflers functional requirements that should be considered for any required application. They tested the performance of an absorptive style muffler for a formula SAE vehicle using exhaust noise measurement with a sound level analyzer. They considered two cases; muffled and unmuffled engine. It was concluded that muffled one provided the required attenuation and produced minimal backpressure.

More optimization work was done by Jin Woo Lee and Yoon Young Kim, 2009 [28].They optimized partition layouts inside expansion chamber of a muffler. They used a formulation acoustical topology optimization problem to increase TL in target frequencies. Also investigated the effects of partition volume constraint ratios, and explained physics behind the optimized layouts. Accepted numerical results of the optimized partition layouts, but shapes and locations of the optimized partitions should be much different from those of conventional partitions. Obtained higher TL values in the target frequencies result from the sacrifice of the TL at other frequencies. They described some results and methods which are expected to be utilized for optimizing 3-D non-concentric expansion chamber mufflers as well as cylindrical symmetric mufflers.Recently, Thomas and Hikkola, 2010 [29] made measurements to adjust reactive muffler components to optimize the shape. The objective of optimization was to maximize sound TL at multiple frequency ranges. Each frequency determined by a hybrid method, which requires solving Helmholtz numerically by FEM. the optimization performed using non-dominated sorting genetic algorithm NSGA-2.

Moreover, a new suggestion to design and optimize mufflers acoustic performance as an overall transmission loss was conducted by Komkin, 2010 [30]. He proposed using a muffler prototype with nondimensional geometrical parameters and integral criterion of acoustic performance of muffler. So, the actual dimensions inlet/outlet being known. That proposed approach enables one to resolve muffler geometry optimization problem. Chamber of muffler with one or two sections was taken as example.Also, Min-Chie Chiu 2008 [31] focused on shape optimization of multi-chamber plug-inlet mufflers along with work on maximal back pressure. He introduced a numerical case for eliminating broadband steam blow-off noise using multichamber plug-inlet mufflers in conjunction with a genetic algorithm (GA) as well as a numerical decoupling technique, all within a space-constrained pressure drop. To verify the reliability of the GA optimization, optimal noise abatements for various pure tones on a one-chamber plug-inlet muffler were examined. The accuracy of the mathematical model was validated using experimental data. Subsequently, optimal results then indicate that the maximal sound transmission losses were indeed located at the desired target tones. The results indicated that both pressure drop and acoustical performance increased when the diameters (at inlet tubes and perforated holes), the perforated ratio, and the length of perforated tubes were decreased.

A parametric optimization to evaluate the appropriate size of extended inlet/outlet duct was employed by Key Fonseca de Lima et al, 2011 [32]. They employed of shape optimization to establish the proper profile of these ducts in order to improve the acoustic features of the muffler in a specific frequency range. The methodology of mixing parametric with shape optimization produces good results of reactive silencer optimization. They defined the objective function used through the average TL for desired frequency range. This type of objective function is strongly non-linear, Genetic algorithm GA is recommended to determine the maximum of this function as a mathematical method. They obtained good results with the GA technique associated with the TL evaluations through the FEM together with the improved four parameter method. In additions, Maddali, 2011[33] conducted parametric studies to understand the effects of various parameters on cylindrically shaped muffler configurations. He obtained a derivation of four-pole parameters of muffler configuration using pressure response function by FEM considering a higher mode effect. Assuming Insertion 
Loss (TL) as a performance parameter, also by offsetting of outlet radically, changing of expansion chamber and inlet dimensions using derived four-pole parameter. It was observed that inlet/outlet pipe would begin to affect IL when they were much longer than the expansion chamber. More number of anti-peaks in the IL plots was observed as the length of the inlet/outlet was increased. However on the whole, Insertion Loss did not change by much with the increase in length of inlet and outlet pipe.

Most recently, Wang in 2013 [18] examined the acoustic characteristics of the muffler under the factors combined effects of flow rate, temperature and thermal viscous effects, to calculate the transmission loss. They used finite element method, the transfer matrix method and experimental analysis. In the entire frequency range, results using FEM calculation are in good agreement with the experimental results. The results showed that using the transfer matrix method only at the low-frequency results are accurate and with the advent of highfrequency mode wave and then using the transfer matrix method for the calculation of the result is not credible and the passing frequency is much in high-frequency domain. Also, the mathematical method using MATLAB software was conducted by Rahul at el. 2014 [1] to study the Transmission loss of the muffler. They concluded that Transmission loss can be increased by adding protrusion pipe at inlet and outlet. It also concluded that the finite element modal analysis has certain significance in the study of vibration characteristics of the muffler. The time required for optimization of muffler using ANSYS and MATLAB was very short and can be repeated simply after changing the input parameters which provides an easy way to find an optimum solution for muffler design.

\section{Conclusion}

In conclusion, even if a large amount of research has been performed and the main sources have been understood, a final solution for muffler noise seems to be far away. The idea of the author was to survey much research work to concentrate on a specific topic in muffler noise performance using Transmission Loss. From in-depth study, it is tried in this paper to give a completereview of the attempts accomplished in this fieldfocusing on different aspects of the subject.Furthermore, to give a general overview on the topicand direct the reader toward practical and applied useof this information, the most important and applicablecomputer codes and some practical results of usingthis technique were also presented.Based on the above review of the literature on automotive muffler Transmission Loss, it is observed that the current work on the optimization of muffler noise is quite desirable and more research need to do for complete optimization.

\section{References}

[1]. Rahul D. Nazirkar, S.R.Meshram, Amol D. Namdas, Suraj U. Navagire, Sumit S. Devarshi "Design \& Optimization Of Exhaust Muffler \& Design Validation" Proceedings of 10th IRF International Conference, 1st June-2014, Pune, India.

[2]. Cheng, C.Y.R. and Seybert, A.F. (1991) "A multidomain boundary element solution for silencer and muffler performance prediction", J. Sound and Vibration, 151, 119-129.

[3]. Selamet, A. and Ji, Z.L. (1999) "Acoustic attenuation performance of circular expansion chambers with extended inlet/outlet", J. Sound and Vibration, 223, 197-212.

[4]. Middelberg, J.M, Barber, T.J., Leong, S.S., Byrne, K.P. and Leonardi, E. (2004) "CFD analysis of the acoustic and mean flow performance of simple expansion chamber mufflers", Proc. ASME Intl. Mechanical Engineering Congress andExposition, Anaheim, California.

[5]. A.K.M.Muhiuddin,Ataur Rahman and Yazid Bin Gazali, "simulation and experimental investigation of muffler performance" international journal of mechanical and materials engineering, Vol2,2007.

[6]. HungguangLiu,SinliLu,Falin Zeng,Zhangping Lu and Jiangkun Zhou, "A study on the effects of higher order mode wave on mufflers performance, modern applied science vol.3 no.1, 2009.

[7]. Mohamed R. SabryAllam, K. A. Abdelgwad, Nouby. M. and ali M. Abdeltwab, "modelling and analysis of single expansion chamber using response surface methodology" international journal of mechanical and materials engineering vol.2 issue 1, 2012.

[8]. Potente, Daniel, "General Design Principles for an Automotive Muffler”, Proceedings of Acoustics, 2005.

[9]. Tyler W. Le Roy "Muffler Characterization with Implementation of the Finite Element Method and Experimental Techniques" Master Of Science, Michigan Technological University, 2011.

[10]. Z.L. Ji "Boundary element analysis of a straight-through hybrid silencer" Journal of Sound and Vibration 292 (2006) 415-423.

[11]. X.LI and C.H.Hansen, "comparison of models for predicting the transmission loss of plenum chambers" applied acoustics 66p.p.810-828,2005.

[12]. K.T.Chen,Y.H.Chen and K.Y.Lin, "The improvement on the transmission loss of a duct by adding Helmholtez resonators" applied acoustics vol.54 no.1 1998 .

[13]. J. Stuart Bolton, TaewookYoo and OlivieroOlivieri,Technical review transmission loss measurements and other acoustical properties of materials placed in standing wave tube,sound and vibration, no.1, 2007.

[14]. S. Bilawchuk and K.R. Fyfe,comparison and implementation of the various numerical methods used for calculating transmission loss in silencer system,Applied Acoustics 64,p.p. 903-916,2003.

[15]. Bilawchuk, S. and Fyfe, K.R. (2003) "Comparison and implementation of the various numerical methods used for calculating transmission loss in silencer systems", Appl.Acoustics, 64, 903-916

[16]. Hyunsu Kim and M.S., transmission loss of silencers with flow from a flow impedance tube using burst signals, OhioUniv. PH.D, 2011.

[17]. FlorentMasson,PabloKogan and Gonzalo Herrera, Optimization of muffler transmission loss by using microperforated panels, VI Congresolberoamericano de Acústica - FIA,2008.

[18]. Wang B., Wang Yongjuan and XU Cheng “ Study of Transmission Loss on Muffler” Research Journal of Applied Sciences, Engineering and Technology 5(24): 5556-5560, 2013 
[19]. Parrot T., An improved method for design of an expansion chamber muffler with application to an operational helicopter, NACA tnd-7309,1973

[20]. Amiya R. and Mohanty, experimental and numerical investigation of reactive and dissipative mufflers,Kentucky univ. PH.D, 1993.

[21]. Long-JyiYeh, Ying-Chun Chang, Min-Chie Chiu and Gaung-Jer Lay,Computer-aided optimal design of a single chamber muffler with side inlet/outlet under space constraints, Marine Science and Technology, 2003.

[22]. Long-JyiYeh, Ying-Chun Chang, and Min-Chie Chiu,application of genetic algorithm to the shape optimization of a constrained double chamber muffler with extended tube,Journal of Marine Science and Technology,Vol. 12, No. 3, pp. 189-199,2004.

[23]. Selamet, I.J. Lee and N.T. Huff, Acoustic attenuation of hybrid silencers, Journal of Sound and Vibration 262 (2003) $509-527$.

[24]. Z.Tao and A.F.Syebert,a review of current techniques for measuring muffler transmission loss, SAE website, 2003.

[25]. E.Besache,R.Ben Fatma,A.S. Bonnet-Bendhia,D. Drissi and N. Gmati,numerical simulation of exhaust muffler-an homogenized finite element method,Volume 1, pages 1- 10, 2006.

[26]. Mihai Bugaru and OvidueVasili,Transfer matrix method for a single-chamber mufflers,applied mathematics international conference 2007.

[27]. Rahul Bansal, experimental techniques for determination of linear and no-linear acoustic properties of perforates, Asia-link asia $111000,2005$.

[28]. Jin Woo Lee and Yoon Young Kim, Topology optimization of muffler internal partitions for improving acoustical attenuation performance, international journal for numerical methods engineering, p.p.455-477, 2009.

[29]. Thomas airaksinen and ErrickHikkola,multiobjective muffler shape optimization with hybrid acoustics modeling,reports of the dep. Mathematical inf. Tech.series B scientific computing No.B6/2010.

[30]. Komkin,I. Optimization of Reactive Mufflers,Acoustical Physics,Vol. 56, No. 3, pp. 336-341, 2010

[31]. Min-Chie Chiu and Ying-Chun Chang,Numerical studies on venting system with multi-chamber perforated mufflers by GA optimization,Applied Acoustics 69, p.p. 1017-1037, 2008.

[32]. Key Fonseca de Lima, ArcanjoLenzi and Renato Barbieri,The study of reactive silencers by shape and parametric optimization techniques,Applied Acoustics 72, pp.142-150,2011

[33]. RamakanthMaddali, parametric studies of circular expansion chambers using four-pole matrix approach while considering higher order mode effects, Master of Science in mechanical engineering, 2011. 